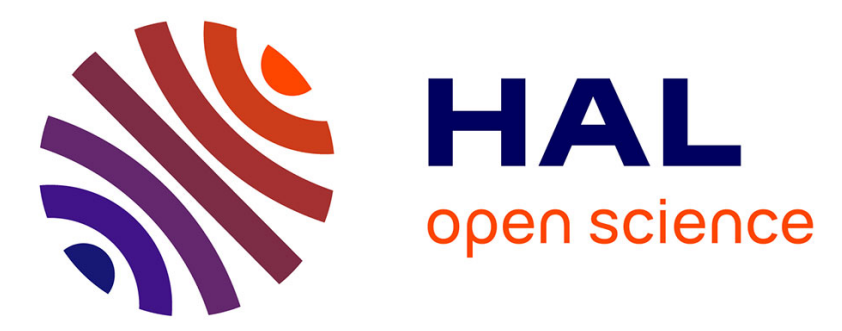

\title{
Techno-economic analysis of MIMO \& DAS in 5G
}

Christos Bouras, Stylianos Kokkalis, Anastasia Kollia, Andreas Papazois

\section{To cite this version:}

Christos Bouras, Stylianos Kokkalis, Anastasia Kollia, Andreas Papazois. Techno-economic analysis of MIMO \& DAS in 5G. 11th IFIP Wireless and Mobile Networking Conference (WMNC 2018), Sep 2018, Prague, Czech Republic. pp.73-80. hal-01995438

\section{HAL Id: hal-01995438 \\ https://hal.inria.fr/hal-01995438}

Submitted on 26 Jan 2019

HAL is a multi-disciplinary open access archive for the deposit and dissemination of scientific research documents, whether they are published or not. The documents may come from teaching and research institutions in France or abroad, or from public or private research centers.
L'archive ouverte pluridisciplinaire HAL, est destinée au dépôt et à la diffusion de documents scientifiques de niveau recherche, publiés ou non, émanant des établissements d'enseignement et de recherche français ou étrangers, des laboratoires publics ou privés.

\section{(c)(1)}

Distributed under a Creative Commons Attribution| 4.0 International License 


\title{
Techno-economic analysis of MIMO \& DAS in 5G
}

\author{
Christos Bouras $^{* \dagger}$, Stylianos Kokkalis ${ }^{\dagger}$, Anastasia Kollia $^{\dagger}$, Andreas Papazois $^{\dagger}$ \\ *Computer Technology Institute \& Press "Diophantus", Patras, Greece \\ ${ }^{\dagger}$ Computer Engineering \& Informatics Dept., University of Patras, Greece \\ bouras@cti.gr, kokkali@ceid.upatras.gr, akollia@ceid.upatras.gr,papazois@ceid.upatras.gr
}

\begin{abstract}
G is going to be put into operation, therefore, it is fundamental that all enabling technologies are analyzed in a technical and economic way. The business sector is not very favorable in investing into novel technologies. In this paper, authors propose a techno-economic model for the Multiple Input Multiple Output (MIMO) technology and compare it to the previously developed model for the Distributed Antenna Systems (DAS). Firstly, the experimentation models are analyzed. Secondly, the mathematical models are presented and the specific parameters are opted. Several experiments help comparing and contrasting the two different technologies. There are not many works concerning the technoeconomic perspectives of MIMO comparing it with the DAS, this research contributes in this direction.
\end{abstract}

Keywords-5G, pricing, cost models, mobile networks, MIMO, Massive MIMO, DAS

\section{INTRODUCTION}

The advent of $5 \mathrm{G}$ coincided with the economic crisis. Telecommunication operators never cease to seek new ways of obtaining profits with little or no investment in novel technologies. The requirements of $5 \mathrm{G}$ are met by different technologies, than nowadays. 5G's realization will include fundamental technological achievements, such as Device to Device communications (D2D) and Internet of Things (IoT), which will augment the number of connected devices to mobile networks. 5G is supposed to reduce latency (less than $1 \mathrm{~ms}$ ), augment the throughput. Therefore, the existing technologies can not keep up with these demands.

Key technologies for $5 \mathrm{G}$, such as Software Defined Networking (SDN), Network Function Virtualization (NFV), Multiple Input Multiple Output (MIMO), cognitive radio, Ultra-density etc. are going to support the development of networks in the future [1]. One of the key technologies of $5 \mathrm{G}$ networks will be MIMO, which is a wireless technology that offers the capability to enhance the rate of the data transfered using a number of antennas. The existing antennas help transmitting the signals into smaller regions of space. There is also a version of the MIMO, which is called Massive MIMO as the number of the included antennas is enlarged. This technology contributes in meeting $5 \mathrm{G}$ requirements, because it offers: [2] increased data rates, enhanced reliability, improved energy efficiency and reduced interference.

An optimal design for Base Stations (BS) is discussed in
[3], where it is pinpointed that MIMO unresolved issues focus on finding the equilibrium among the appropriate bandwidth (BW) needed, the quantization number in bits and the number of antennas needed to maximize the sum of achievable rates. MIMO technology has been thoroughly researched in [4], while in terms of performance MIMO has been investigated in [5] and [6]. When it comes to cost analysis, the main existing study [7] compares MIMO with the Licensed Shared Access (LSA) in Finland. There are several cost analyses concerning 5G networks [8].

Authors have already presented research concerning the techno-economics of $5 \mathrm{G}$ technologies. They have already developed ultra-dense and DAS techno-economic models [9], Sensitivity Analysis (SA) of DAS and small cells [10], [11]. In previous research, they have presented cost models for SDN and NFV [12] and they have also thoroughly analyzed the SDN and NFV concepts in mobile networks alongside with the advancements and challenges they induce [13].

In this research, MIMO and DAS will be compared. MIMO and DAS are two different technologies, they are both based on a number of antennas that enable connectivity with different terminals in the network. They could be used in $5 \mathrm{G}$ networks. In future mobile networks, antennas are added in the network to augment its capacity and coverage and rend it more efficient. To our knowledge there is not a technoeconomic comparison between these two technologies.

The remaining part of this paper is structured as follows: In Section II the proposed architectures are analyzed and explained. In Section III the proposed financial and mathematical models are summarized. In Section IV the experimentation parameters are opted and explained thoroughly. In Section $\mathrm{V}$ several experiments concerning the models' viability are conduced. In Section VI conclusions are summarized and future research activity in the field is proposed.

\section{ARCHITECTURE}

In this section, MIMO and DAS technologies are presented.

\section{A. Multiple Input Multiple Output (MIMO)}

MIMO is a technology inextricably connected with antennas. In this technological concept, several antennas exist 


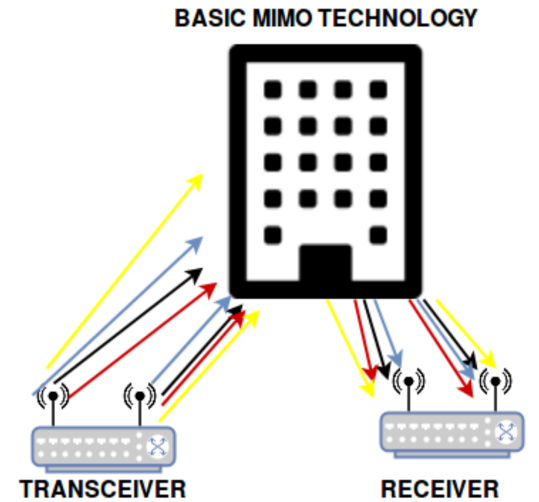

Fig. 1: The MIMO technology generic concept.

both on the side of the transceiver and the side of the receiver. This technique sets as principal goal the enhancement of the coverage, data rates and reduction of the latency in a wireless or mobile network. What is more, smart antenna technology is exploited and used within MIMOs electronic circuits in order to minimize errors and augment speed. Nowadays, MIMO is a well-known technology used for the BS, starting with the 4th generation of mobile networks, but in a very different aspect. Common forms of the simple MIMO include $4,8,16$ antennas at the transceiver's and the receiver's sides. These numbers could differ for the different sides.

Fig. 1 depicts the general concept of a MIMO architecture. $N$ stands for the total numbers of antennas existing in the scheme. When the number of antennas is $4,8,16$, then the technology is called MIMO. When there is a huge number of antennas in each transceiver or receiver e.g. 128, 256, etc. then the scheme is called Massive MIMO and it is claimed to be one of the main technologies for the 5th generation of mobile networks, as it promises all the above mentioned fundamental advantages.

\section{B. Distributed Antenna System (DAS)}

DAS was thoroughly analyzed in previous research activity, such as [10], [11]. It includes a number of antenna nodes connected in a transmission medium, e.g. optic fiber. It is possible to combine it with 802.11 and even enhance it. In previous research activity [9], it was indicated that two antennas per floor of a building could sufficiently cover the floor of a block of flats or an under-populated building. But, when it comes to larger places, several sets of antennas need to be added to succeed in covering the whole place. There are several substantial benefits of this solution (e.g. high coverage, power efficiency, scalability etc.) and this is why it is supposed to be a good alternative for $5 \mathrm{G}$ networks.

The DAS includes two basic structures. There is a BS and a Distributed System (DS). A number of antennas, as mentioned before represents the DS alongside with a number of transmitters and feeders that facilitate the transmission. A

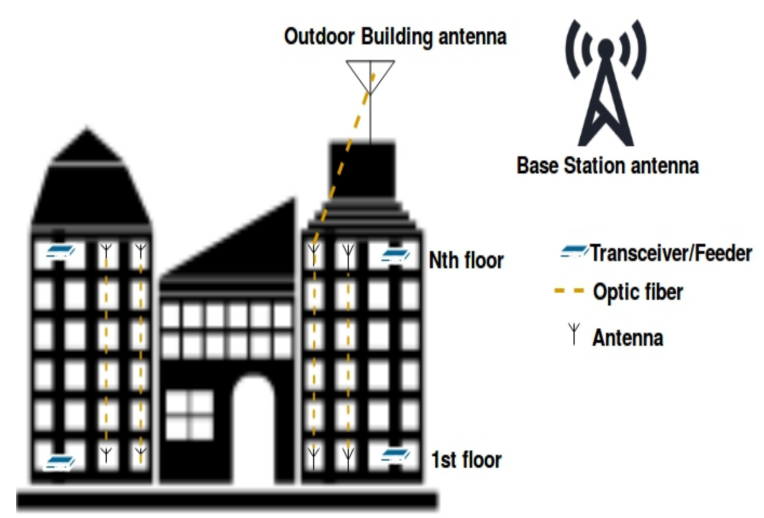

Fig. 2: A model for in-building conventional DAS architectures.

transmission medium is needed and optic fiber is the one that is opted, because it offers lower losses.

Fig. 2 depicts a basic architectural model for the DAS structure. It includes several antennas per floor (at least two), a number of transceivers and feeders in each floor. All equipment is connected to the optic fiber, which is lead to the outdoor antenna at the top of the building, which exchanges information with the BS antenna and enables the communication between the building's devices and the outer world.

\section{PRICING MODEL}

In this section, the pricing models developed for the techno-economic comparison are analyzed.

In general, the Total Cost of Ownership (TCO) of a technology could be split into two main categories of expenditures:

- the Capital (CAPEX): is all the money spent for the acquisition of the new equipment, site, and it is actually an initial investment in a technology, product or service and

- the Operational (OPEX): represents the budget needed on a defined recurring basis, such as maintenance, operation costs, such as power consumption costs etc.

These two different cost categories are really deviant and therefore, a specific methodology followed in [9] could help in forming both costs. The annual cost estimation is achieved by considering that the CAPEX as an investment, whose capital was obtained through a loan. Afterwards, the corresponding annual cost results by calculating the annual installment payments that correspond to the repayments of this loan.

Supposedly that $P$ is the amount of money spent for the loan and is repaid annually, the repeating payment formula 
could be used and the installment payment would be:

$$
A=P \frac{r(1+r)^{n}}{(1+r)^{n}-1}
$$

where $r$ represents the periodic interest rate and $n$ represents the length of the installment plan in years.

\section{A. Multiple Input Multiple Output (MIMO)}

1) Capital Expenditure: CAPEX is the amount of money invested for the deployment of the new infrastructure and/or the acquisition of the new equipment. CAPEX also includes the investments made on updating the infrastructure with new hardware, building, installation, back-hauling etc. The installation costs for the BS nodes alongside with the core network therefore, appertain to this cost category.

If $C_{e N B}$ corresponds to the cost of a BS and $C_{E P C}$ is the cost for the amendments and installations made for the Evolved Packet Core (EPC), namely the key terms used into the LTE-A, then the cost for a BS will be given by the expression: : $C_{e N B}+C_{E P C}$. If for example $B$ BS exist in the network, then the cost is formated as $B\left(C_{e N B}+C_{E P C}\right)$. $C_{e N B}$ includes all costs of acquiring the site, back-hauling and construction. $C_{E P C}$ includes all the costs related to the core network.

It is substantial that the cost of the added antennas on both sides is calculated. $M_{s}$ and $M_{d}$ should be the number of antennas in the source and in the destination respectively. $M_{s}+M_{d}$ is the total number of existing antennas per network component.

So, the CAPEX for MIMO is: $B\left(C_{e N B}+C_{E P C}\right)\left(M_{s}+\right.$ $\left.M_{d}\right)$ and as the CAPEX is an investment amount, using the (1) the total CAPEX is given by:

$$
C_{M I M O}^{C X}=B\left(C_{e N B}+C_{E P C}\right)\left(M_{s}+M_{d}\right) \frac{r(1+r)^{n}}{(1+r)^{n}-1}
$$

where $C_{M I M O}^{C X}$ denotes the annual CAPEX, and $n$ is the installment plan in years.

2) Operational Expenditure: OPEX includes the costs for the network's day-to-day operation and maintenance activities. There are costs for power, in and off site support and maintenance, which are represented by the coefficient $c_{r u n}$ and back-hauling is denoted by $c_{b h}$. For the $B$ existing BS these costs are given by: $B\left(c_{\text {run }}+c_{b h}\right)$. Considering the MIMO antennas as well, then the cost is formed as: $B\left(c_{\text {run }}+c_{b h}\right)\left(M_{s}+M_{d}\right)$.

Site, operation and support costs could be expressed by $c_{s t}$. In order to include the costs paid for all the base stations, $B c_{s t}$ represents this type of cost. The needed BW $B W$ multiplied with a coefficient $f_{B W}$ denotes the cost for the BW leasing and back-hauling needed.

To summarize, the OPEX for the MIMO is:

$$
\begin{aligned}
C_{M I M O}^{O X}= & \left(M_{s}+M_{d}\right)\left[B\left(c_{r u n}+c_{b h}\right)\left(C_{e N B}+C_{E P C}\right)\right. \\
& \left.\left.\frac{r(1+r)^{n}}{(1+r)^{n}-1}+B c_{s t}+f_{B W} B W\right)\right]
\end{aligned}
$$

3) Total Cost of Ownership: The TCO of a network is the sum of the CAPEX and OPEX of all its components, therefore:

$$
\begin{aligned}
c_{M I M O}^{T C O}= & B\left(C_{e N B}+C_{E P C}\right)\left(M_{s}+M_{d}\right) \frac{r(1+r)^{n}}{(1+r)^{n}-1}+ \\
& \left(M_{s}+M_{d}\right)\left[B\left(c_{r u n}+c_{b h}\right)\left(C_{e N B}+C_{E P C}\right)\right. \\
& \left.\frac{r(1+r)^{n}}{(1+r)^{n}-1}+B c_{s t}+f_{B W} B W\right]
\end{aligned}
$$

where $i$ is the interest rate and $n$ is the duration of the installment plan expressed in years.

\section{B. Distributed Antenna System (DAS)}

The pricing model for the DAS has already been described in previous research activity and therefore, in this paper it is going to be summed up.

\section{CAPEX}

DAS' CAPEX consists of the costs for obtaining the novel infrastructure and the actions for integrating new ones when the existing equipment is updated. DAS BS costs are given by the following equation: $C_{e N B}+C_{E P C}$. BS is represented by the coefficient $C_{e N B}$ and $C_{E P C}$ represents the costs for the EPC. If there are $N$ BS, then $N\left(C_{e N B}+C_{E P C}\right)$ is the cost for all the BS. As this amount is repaid annually 1 , then DAS BS is given by:

$$
C_{B S}^{C X}=N\left(C_{e N B}+C_{E P C}\right) \frac{r(1+r)^{n}}{(1+r)^{n}-1}
$$

$i$ denotes the interest rate and $n$ the installment plan in years.

$C_{e q}$ represents the costs for the DAS equipment. If there are $d$ DSs then the CAPEX for the DS per year is:

$$
C_{D A S E Q}^{C X}=C_{e q} d \frac{r(1+r)^{n}}{(1+r)^{n}-1}
$$

where $C_{D A S E Q}^{C X}$, denotes the annual total cost of DS equipment CAPEX. Implementation costs $C_{i n c}$ represent the costs for integrating and installing the new equipment.

In order to calculate the total CAPEX, then:

$C_{D A S}^{C X}=\left(C_{e q} d+N\left(C_{e N B}+C_{E P C}\right)\right) \frac{r(1+r)^{n}}{(1+r)^{n}-1}+C_{i n c}$

$C_{D A S}^{C X}$ represents the CAPEX yearly.

\section{OPEX}

OPEX represents all the costs concerning the day-today operation of the system. It also includes the power consumption costs, leasing, site etc. All these costs are represented by the $c_{\text {run }}$. The back-hauling is represented by the coefficient $c_{b h}$. The DAS BS OPEX costs on a yearly basis is:

$$
C_{B S D A S}^{O X}=N\left(c_{\text {run }}+c_{b h}\right)
$$


$C_{e q}$ denotes the costs for the DS and $d$ is the numbers of the DS in the building. The OPEX for all the DS is:

$$
C_{D S D A S}^{O X}=C_{e q} d \frac{r(1+r)^{n}}{(1+r)^{n}-1}
$$

The power consumption for the operation of the system is denoted by $C_{p w}$. The total $\mathrm{BW}$ is given by the coefficient $B W$ multiplied with a coefficient, that denotes the backhauling activities for the $\mathrm{BW}$, namely the $f_{B W}$. Therefore, the OPEX of the DAS is given by:

$$
\begin{aligned}
C_{D A S}^{O X}= & N\left(c_{\text {run }}+c_{b h}\right)+f_{B W} B W+ \\
& +\left(f_{s t}+C_{p w} C_{e q} d\right) \frac{r(1+r)^{n}}{(1+r)^{n}-1}
\end{aligned}
$$

\section{E. TCO} and 8:

The TCO of the DAS model is given by the sum of 7

$$
\begin{array}{r}
T C O_{D A S}=\left(N\left(C_{e N B}+C_{E P C}\right)+C_{e q} d\left(1+C_{p w}\right)+f_{s t}\right) \\
\frac{r(1+r)^{n}}{(1+r)^{n}-1}+C_{i n c}+N\left(c_{r u n}+c_{b h}\right)+f_{B W} B W
\end{array}
$$

\section{PARAMETER SELECTION}

In this section, the parameters of the proposed models are opted. In order to conduct the experiments and conclude to the influence of the cost parameters in the overall model concerning that the operators will be encouraged or discouraged to invest in this technology, it is crucial that a specific test bench with appropriate parameter values are opted. Values for the network components of the Greek Market are available, because of previous research activity [9]. 5G is going to be realized in the future, it is fundamental to make a provision concerning, what the prices will be. The values of the prices vary within a price range of $+/-50 \%$ of today's value. It is probable that in the next few years prices will be augmented, but on the other hand, the integration of novel technologies into networks, (e.g. SDN and NFV), could offer important advantages and reduce the costs for network components. Virtualization is supposed to reduce the costs $20-80 \%$.

Table I includes all the cost parameters, that are opted for the MIMO scenarios.

In order to complete the experimental procedure, SA will be used. SA is a strong tool, which will be used to indicate how components affect the model. It helps pinpointing the factors that should be diminished for the network to be effective and profitable to the operator. SA is met in twodifferent types:

- One way SA: When one of the parameters of the problem ranges within the price range and the outcome of this reduction/augmentation is described. $B$ $N, n, r, C_{s} t, C_{r} u n, C_{b} h$.

- Multi-way SA: When two or more of the parameters of the problem range within the price ranges and the outcome of the corresponding fluctuations is described. $M_{s} \& M_{d}, C_{e N B} \& C_{E P C}$ and $B W \&$ $f_{B W}$.

\section{EXPERIMENTAL PROCEDURE}

In this section, the experiments are conducted. In the Flow Chart 1 the procedure followed for the experiments in this research is summarized. Different parameters affect the TCO of the model in a different way, SA is realized for all the components of the model. There are several parameters, that refer to the same component of the network.

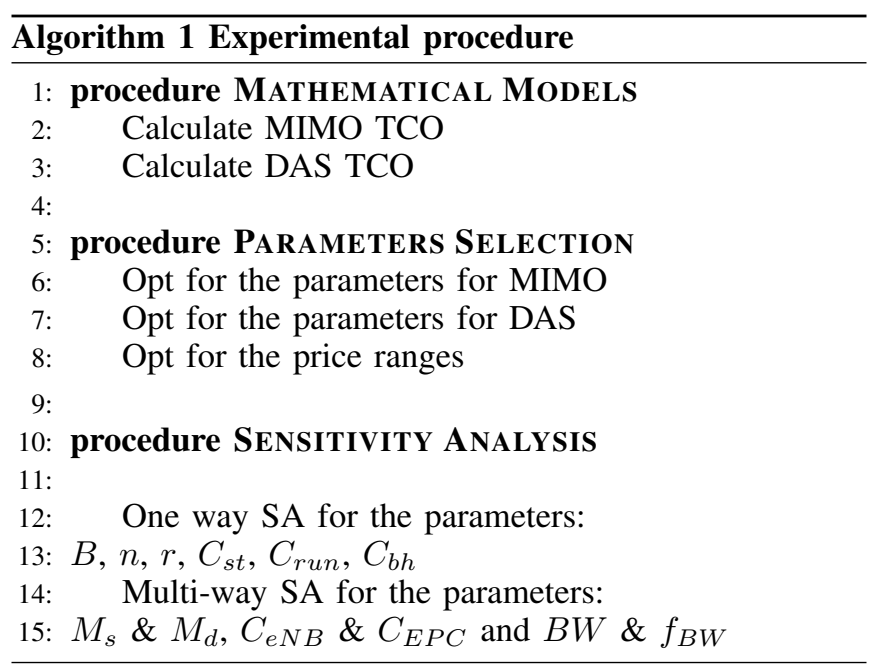

\section{A. Comparison of MIMO \& DAS}

In this section, there will be several experiments that will help comparing and contrasting the two selected technologies.

Fig. 3 depicts the comparison for the CAPEX of the two models. For the MIMO cases (number of antennas $\leq 64$ ) the CAPEX does not diverge compared to the CAPEX of DAS. CAPEX for Massive MIMO (number of antennas $\geq$ 64 ) is exponentially augmenting in relation to the number of antennas. The DAS CAPEX increases in a much slower pace, when the number of added antennas exceeds 1000. Fig. 4 depicts the comparison of the OPEX of the two models. For the MIMO cases (number of antennas $\leq 64$ ) the OPEX does not diverge compared to the one of DAS. OPEX for Massive MIMO (number of antennas $\geq 64$ ) is exponentially augmenting in relation to the number of antennas. The DAS OPEX seems stable for the antennas added. Fig. 5 depicts the comparison of the TCO of the two models. For the MIMO cases (number of antennas $\leq 64$ ) the TCO does not diverge compared to the one of DAS. The TCO for Massive MIMO (number of antennas $\geq 64$ ) is exponentially augmenting in relation to the number of antennas. The DAS TCO increases in a much slower pace, when the number of added antennas exceeds 1000 . 
TABLE I: TCO Cost Parameters and System Variables.

\begin{tabular}{|c|c|c|c|}
\hline \multicolumn{4}{|c|}{ General Costs } \\
\hline Parameter & Description & Value [9] & Value Range for SA \\
\hline$C_{e N B}$ & Capital cost for a single BS & $1000 €[9]$ & {$[500,1500]$} \\
\hline$C_{E P C}$ & $\begin{array}{l}\text { Core network's capital cost for the deployment } \\
\text { of a single eNB }\end{array}$ & $110 €[9]$ & {$[55,165]$} \\
\hline$B / N$ & The total number of BS's and EPC's needed & $1[9]$ & {$[1,100]$} \\
\hline$n$ & Duration of installment plan of a site in years & $10 \mathrm{yrs}$ & {$[5,20][9]$} \\
\hline$r$ & Periodic interest rate & $6 \%[9]$ & {$[2,10]$} \\
\hline$c_{s t}$ & $\begin{array}{l}\text { Site costs apart from maintenance cost, e.g., } \\
\text { power, in-site and off-site support }\end{array}$ & $3100 €[9]$ & {$[1150,4650]$} \\
\hline$C_{\text {run }}$ & Running costs, such as single site, in-site, off-site & $892.50 €[9]$ & {$[446.25,1338.75]$} \\
\hline$C_{b h}$ & Backhaul costs for optic fiber & $4800 €[9]$ & {$[2400,7200]$} \\
\hline$B W$ & Backhaul BW for a site's interconnection & 10 Gbps [9] & {$[5,15]$} \\
\hline$f_{B W}$ & $\begin{array}{l}\text { Linear coefficient correlating site annual back- } \\
\text { haul costs with provided BW - expressed in } \\
€ / G b p s\end{array}$ & $1170[9]$ & {$[585,1755]$} \\
\hline \multicolumn{4}{|c|}{$\begin{aligned} \text { MIMO Costs } \\
\end{aligned}$} \\
\hline Parameter & Description & Value [9] & Value Range \\
\hline$M_{s}$ & $\begin{array}{l}\text { Factor related to the MIMO antennas at the } \\
\text { source }\end{array}$ & 64 & {$[2,4,8,16,64,128,256,512]$} \\
\hline$M_{d}$ & $\begin{array}{l}\text { Factor related to the MIMO antennas at the } \\
\text { destination }\end{array}$ & 4 & {$[2,4,8,16,64,128,256,512]$} \\
\hline \multicolumn{4}{|c|}{$\begin{aligned} & \text { DAS Costs } \\
&\end{aligned}$} \\
\hline Parameter & Description & Value [9] & Value Range \\
\hline$C_{e q}$ & DAS equipment & $11900 €$ & {$[5950,17850]$} \\
\hline$d$ & Number of DS & 2 antennas/floor & {$[2,4,8,16,64,128,256,512]$} \\
\hline$f_{s t}$ & Site maintenance costs & 0.8 & 0.8 \\
\hline$C_{p w}$ & Energy consumption costs & $157.68 €$ & {$[78.84,236.54] €$} \\
\hline$C_{i n c}$ & Implementation costs & $2800 €$ & {$[1400,4200] €$} \\
\hline
\end{tabular}

CAPEX COST COMPARISON OF MIMO AND DAS

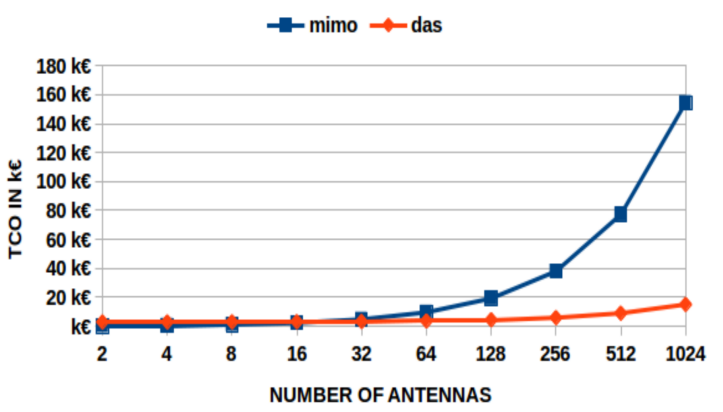

Fig. 3: The comparison of the capital expenditures of MIMO and DAS.

\section{B. Sensitivity Analysis of MIMO costs}

SA indicates which of the components affect the model, therefore, several studies should be developed in the direction of finding technologically efficient approaches to reduce the

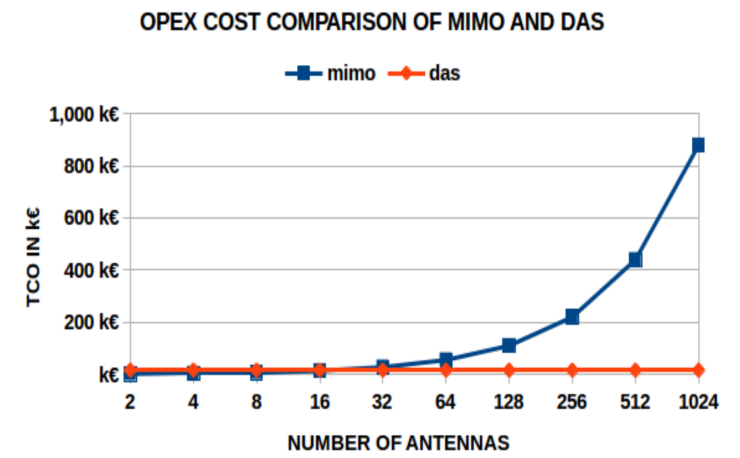

Fig. 4: The comparison of the operational expenditures of MIMO and DAS.

components' costs alongside with maintaining high quality. One-way SA is followed for the parameters that are considered independent from others and it is pursued to show how they affect the CAPEX, OPEX and TCO of the model. On 
TCO COST COMPARISON OF MIMO AND DAS

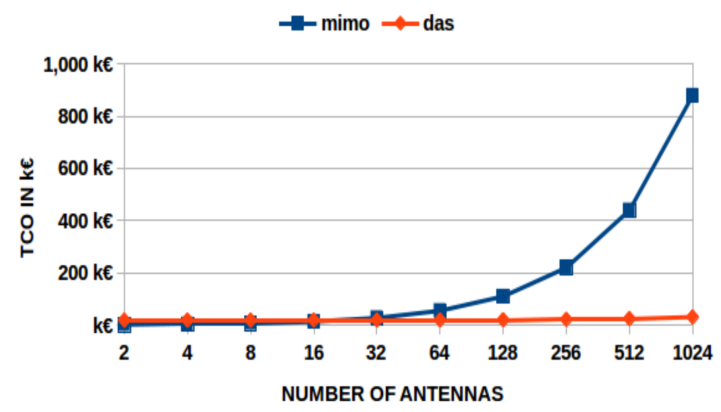

Fig. 5: The comparison of the total cost of ownership of MIMO and DAS.

SENSITIVITY ANALYSIS FOR THE NUMBERS OF BS

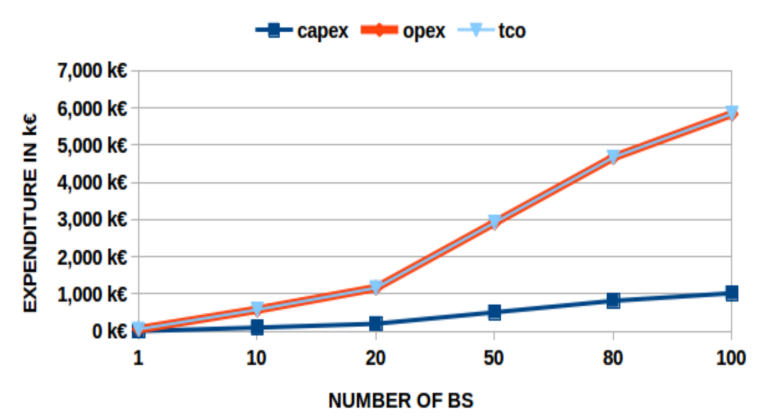

Fig. 6: One-way SA for the number of BS of the MIMO.

the other hand, two-way SA is followed for the parameters that seem to be strongly correlated and/or represent the same network component.

1) One-way Sensitivity Analysis: Fig. 6 indicates that the number of BS affects mainly the OPEX and the TCO of the overall model. All types of costs (CAPEX, OPEX, TCO) are proportional to the number of added antennas, but the CAPEX augments in a lower rate. The OPEX and TCO are also augmenting proportionally to the number of added antennas, but in a much higher rate, which really affects the cost of the model.

Fig. 7 indicates that the number of the investment plan affects all the expenditures. All types of costs (CAPEX, OPEX, TCO) are inversely proportional to the years of the investment, but the CAPEX decreases in a lower rate. The OPEX and TCO are also cut down on inversely to the years of investing in the technology, but in a much higher rate, which really reduces the cost of the model with the elapsing of years. It is therefore obvious that a long-term installment plan is highly affecting the costs and reduces it. The main issue that emerges here is that although mobile network technologies really have low lifetimes, the clientle
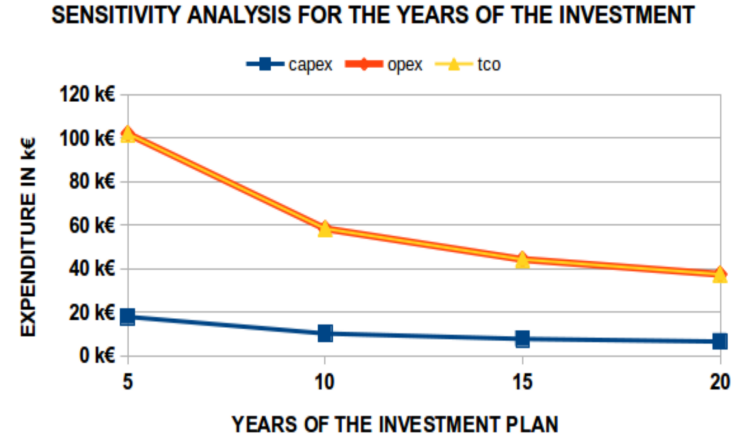

Fig. 7: One-way SA for the years of the investment plan for the MIMO.

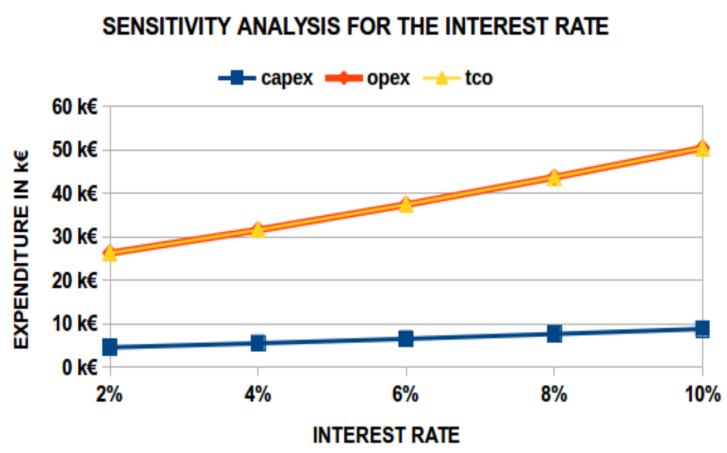

Fig. 8: One-way SA for the interest rate for the MIMO.

raises the market demands and pushes to the development of new products and services. As a result, telecommunication operators seem rather unwilling to invest in a technology, which will have become obsolete in the short future.

Fig. 8 indicates that the interest rate affects mainly the OPEX and the TCO of the overall model. All types of costs (CAPEX, OPEX, TCO) are proportional to the percentage of the interest rate, but the CAPEX augments in a lower pace. The OPEX and TCO are also augmenting proportionally to the interest rate, which really affects the cost of the model. It is therefore obvious that the augmentation of the interest rate is really affecting the costs of the model. Thus, it seems that the prices of the model's components are strongly related to the condition of the market and the banking system at the period of the investment.

Fig. 9 indicates that the site costs affect mainly the OPEX and the TCO of the overall model, because these costs cost more. On the other hand, for all the different prices in the data range of the site costs, the costs are almost stable and therefore, it seems that a certain augmentation or reduction of this component will not highly affect the overall model.

Fig. 10 indicates that the running costs affect mainly the 
SENSITIVITY ANALYSIS FOR THE SITE COSTS

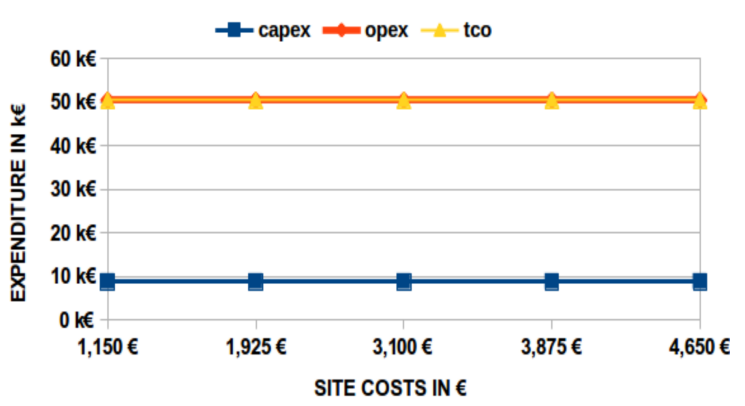

Fig. 9: One-way SA for the MIMO site costs.

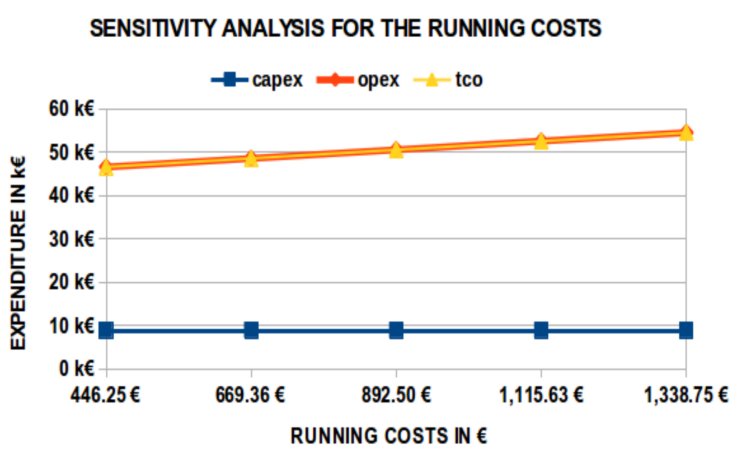

Fig. 10: One-way SA for the MIMO running costs.

OPEX and the TCO of the overall model. OPEX and TCO are proportional to the augmentation of the running costs, but the CAPEX is stable for all the different prices in the data range of the running costs. It is therefore obvious that the augmentation of the running costs is highly affecting the operational costs of the model. Running costs include types of costs related to the operation of the system and all activities related to it. Therefore, a possible divergence in this type of costs is affecting the OPEX and therefore the TCO.

Fig. 11 indicates that the costs for back-hauling affect mainly the OPEX and the TCO of the overall model. OPEX and TCO are proportional to the augmentation of the backhauling costs, but the CAPEX is stable for all the different prices of the back-hauling costs. It is therefore obvious that the augmentation of the back-hauling costs is highly affecting the operational costs of the model. Back-hauling costs include types of costs related to back-haul the system. Therefore, a possible divergence of this type of costs is affecting the OPEX and therefore the TCO. It becomes obvious that in order to maintain low costs of the TCO of the MIMO, a very careful initial design of the model alongside with usage of novel highly-efficient and low-cost technologies for the back-hauling activities should take place.

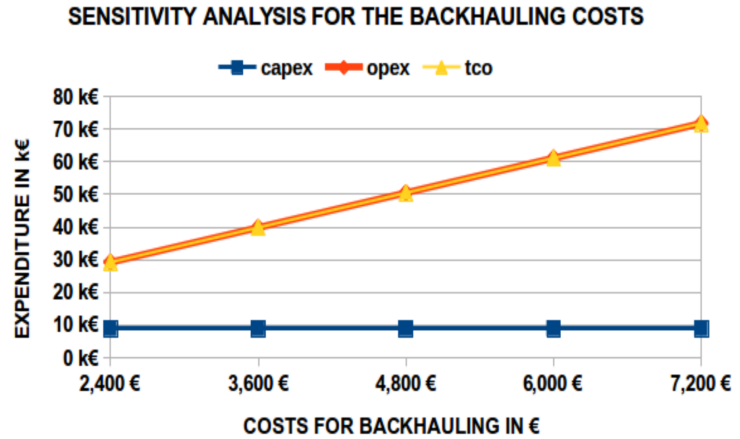

Fig. 11: One-way SA for the MIMO back-hauling costs.

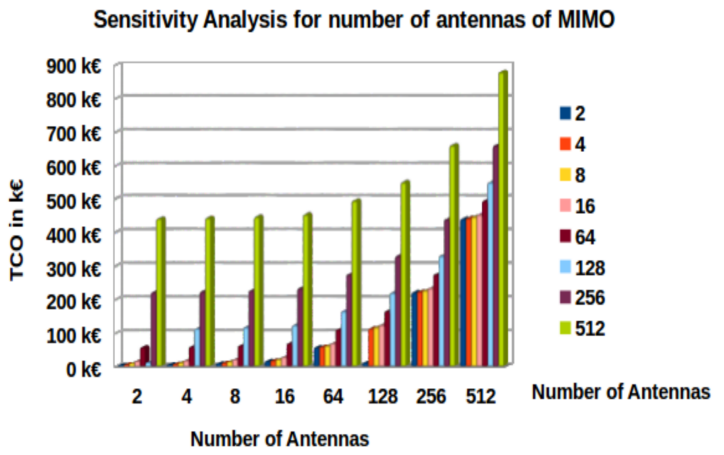

Fig. 12: Two-way SA for the number of antennas of MIMO.

2) Two-way Sensitivity Analysis: Two-way SA is a technique that shows how the costs of a technology are diverging, when a specific set of cost parameters fluctuates within a certain data range. Its basic goal is to indicate, which combination of the cost parameters need to be reduced so that the model becomes cost efficient and widely adopted.

Fig. 12 indicates how the two parameters of the number of antennas are affecting the TCO. It seems that in architectures, that the number of antennas is $\geq 64$, the TCO is really augmented and it may be inefficient. Solutions, that have different number of antennas on each side, with the one side including small numbers of antennas and the other side including high numbers seem to be cost efficient and maybe solving the coverage and connectivity problems. Another question is also raised: Are there any other ways to reduce the antennas' costs? Or is there a more efficient way to use the MIMO concept?

Fig. 13 indicates that the reduction of BS costs leads to lower TCO. Novel ways of adding novel technologies or virtualization methods could help reducing the costs of the BS even more and as a result, to reduce the overall cost of the model.

Fig. 14 indicates that the reduction of $\mathrm{BW}$ costs leads 


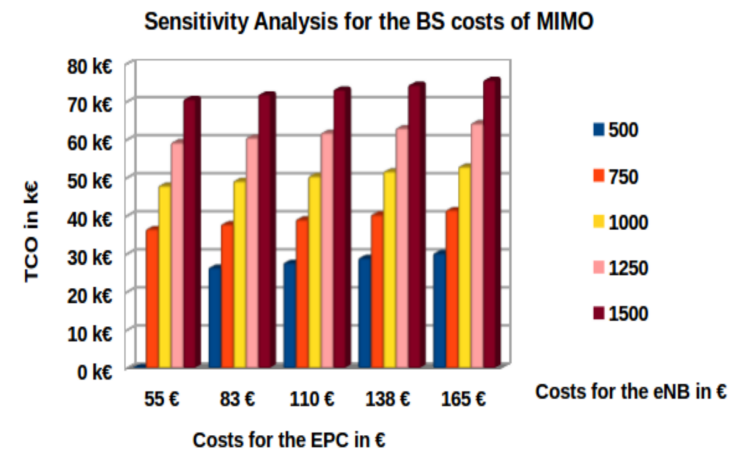

Fig. 13: Two-way SA for the BS costs of MIMO.

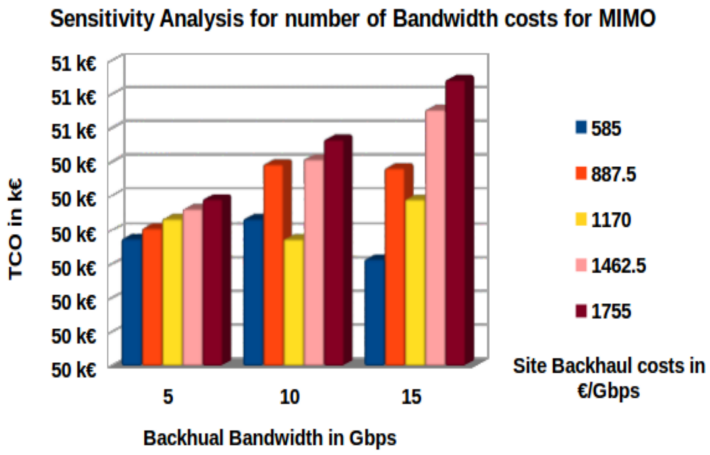

Fig. 14: Two-way SA for the BW costs of MIMO.

to lower TCO. Leasing BW should be better negotiated, also telecommunication companies could push for the diminishing of the BW costs. Investigation of solutions that offer re-usage and better allocation of the BW resources is crucial.

\section{CONCLUSions \& Future Work}

In this paper, MIMO and DAS techno-economic models were compared and contrasted. Both technologies seem promising solutions for the $5 \mathrm{G}$ requirements. On the other hand, their high costs rend it difficult for telecommunication enterprises to turn to them. According to the analysis developed in this paper, it seems that DAS is cheaper than MIMO for large scale applications, but on the other hand, smaller solutions MIMO seem to be more cost efficient. SA led to the fact that operational expenses, such as running costs and power consumption should be reduced to ensure the cost efficiency of the MIMO models.

Future research activity should focus on ways to diminish operational expenditures and running costs. Innovation should be used for the technologies in order to succeed in providing cheaper services for the BS. Reusing BW resources is an open investigation issue. The optimization of MIMO antennas on each side will lead to a high performance and low-cost solution, which is of high significance before the wide adoption of the MIMO and Massive MIMO.

\section{REFERENCES}

[1] I. F. Akyildiz, S. Nie, S.-C. Lin, and M. Chandrasekaran, " $5 \mathrm{~g}$ roadmap: 10 key enabling technologies," Computer Networks, vol. 106, pp. 17-48, 2016.

[2] E. G. Larsson, O. Edfors, F. Tufvesson, and T. L. Marzetta, "Massive mimo for next generation wireless systems," IEEE Communications Magazine, vol. 52, no. 2, pp. 186-195, 2014.

[3] K. Senel, E. Björnson, and E. G. Larsson, "Optimal base station design with limited fronthaul: Massive bandwidth or massive mimo?," arXiv preprint arXiv:1709.05172, 2017.

[4] F. Verde, Y.-W. P. Hong, D. Samardzija, R. Schober, and Z. Tao, "Cooperative mimo multicell networks," EURASIP Journal on Advances in Signal Processing, vol. 2012, no. 1, p. 41, 2012.

[5] Y.-G. Lim, C.-B. Chae, and G. Caire, "Performance analysis of massive mimo for cell-boundary users," IEEE Transactions on Wireless Communications, vol. 14, no. 12, pp. 6827-6842, 2015.

[6] R. Muharar and J. Evans, "Performance analysis of massive mimo networks with random unitary pilot matrices," arXiv preprint arXiv:1709.03325, 2017.

[7] M. Katsigiannis, A. Basaure, and M. Matinmikko, "Cost comparison of licensed shared access (lsa) and mimo scenarios for capacity growth in finland," in $5 G$ for Ubiquitous Connectivity (5GU), 2014 1st International Conference on, pp. 291-296, IEEE, 2014.

[8] W. Elmannai and K. M. Elleithy, "Cost analysis of 5th generation technology," 2014.

[9] C. Bouras, V. Kokkinos, A. Kollia, and A. Papazois, "Technoeconomic analysis of ultra-dense and das deployments in mobile $5 \mathrm{~g}$," in 2015 International Symposium on Wireless Communication Systems (ISWCS), pp. 241-245, Aug 2015.

[10] C. Bouras, A. Kollia, and A. Papazois, "Sensitivity analysis of small cells and das techno-economic models in mobile 5g," in 2016 IEEE Wireless Communications and Networking Conference, pp. 1-6, April 2016.

[11] C. Bouras, A. Kollia, and A. Papazois, "Dense deployments and das in 5g: A techno-economic comparison," Wireless Personal Communications, vol. 94, no. 3, pp. 1777-1797, 2017.

[12] C. Bouras, P. Ntarzanos, and A. Papazois, "Cost modeling for $\mathrm{sdn} / \mathrm{nfv}$ based mobile $5 \mathrm{~g}$ networks," in 2016 8th International Congress on $\mathrm{Ul}$ tra Modern Telecommunications and Control Systems and Workshops (ICUMT), pp. 56-61, Oct 2016.

[13] C. Bouras, A. Kollia, and A. Papazois, "Sdn nfv in 5g: Advancements and challenges," in 2017 20th Conference on Innovations in Clouds, Internet and Networks (ICIN), pp. 107-111, March 2017. 